\title{
High Speed Imaging of Flame Structure and Dynamic Processes in Swirl Stabilized Prevaporized Liquid Fuel Flames
}

\author{
Christoph M. Arndt ${ }^{1, *}$, Adam M. Steinberg ${ }^{2, \dagger,}$, Jan Böhnke $^{3, *}$, Redjem Hadef $^{4, \$}$, Wolfgang Meier ${ }^{5, *}$ \\ *German Aerospace Center (DLR), Institute of Combustion Technology, Stuttgart, Germany \\ ${ }^{\dagger}$ University of Toronto, Institute for Aerospace Studies, Toronto, Canada \\ ${ }^{\S}$ Present Address: Georgia Institute of Technology, Daniel Guggenheim School of Aerospace Engineering, \\ Atlanta, GA, USA \\ ثUniversité Larbi Ben M’Hidi, Institut de Génie Mécanique, Oum El Bouaghi. Algeria
}

\begin{abstract}
Detailed optical measurements with high spatio-temporal resolution were carried out in a gas turbine model combustor (GTMC) at atmospheric pressure using prevaporized ethanol as fuel. The GTMC features co-annular swirl nozzles with separate air plenums for the inner and outer air streams, allowing control of the air split ratio $L$ between the nozzles. In the current study, the air split ratio was fixed at $L=1.6$, which resulted in equal pressure drop across both nozzles. The total air mass flow rate was fixed at $400 \mathrm{~g} / \mathrm{min}$ and flames with different fuel flow rates - resulting in thermal powers between $10.9 \mathrm{~kW}$ and $16.9 \mathrm{~kW}$ and corresponding global equivalence ratios between $\varphi=0.55$ and $\varphi=0.85$ - were studied. Air, fuel, and all supply lines, were preheated to $>100^{\circ} \mathrm{C}$ in order to avoid fuel condensation within the supply lines or the fuel plenum. The flow field was measured with stereoscopic particle image velocimetry (S-PIV), the distribution of the $\mathrm{OH}$ radical within the combustion chamber was measured using planar laser-induced fluorescence (OH PLIF) and the overall flame shape and flame dynamics were imaged using $\mathrm{OH}^{*}$ chemiluminescence $\left(\mathrm{OH}^{*} \mathrm{CL}\right)$. All measurements were performed at a sustained repetition rate of $10 \mathrm{kHz}$. In addition, pressure fluctuations in both air plenums and in the combustion chamber were recorded at a sampling rate of $100 \mathrm{kHz}$ using calibrated microphone probes. All studied flames stabilized within the inner air nozzle, with the main reaction zone being lifted off the burner nozzle. Dominant frequencies for the examined operating conditions were studied by means of fft-analysis of the pressure and heat release rate fluctuations. Furthermore, spatially resolved fft-analysis of the $\mathbf{O H}$ PLIF signal allowed to identify the regions were specific modes are dominant.
\end{abstract}

\footnotetext{
${ }^{1}$ Research Associate, Combustion Diagnostics Department, christoph.arndt@dlr.de

${ }^{2}$ Associate Professor, Senior Member AIAA

${ }^{3}$ Graduate Student, Combustion Diagnostics Department

${ }^{4}$ Professor

${ }^{5}$ Department Head, Combustion Diagnostics Department
} 


\section{Nomenclature}

$L \quad=$ air split ratio

$\dot{m} \quad=$ mass flow

$P_{t h} \quad=$ thermal power

$\varphi \quad=$ equivalence ratio

$\xi \quad=$ mixture fraction

AtJ $=$ alcohol-to-jet

$\mathrm{CL}=$ chemiluminescence

DPSS $=$ diode-pumped solid state

$\mathrm{fft}=$ fast fourier transform

$\mathrm{GT}=$ gas turbine

GTMC = gas turbine model combustor

HRR $=$ heat release rate

IRO $=$ intensified relay optics

$\begin{array}{ll}\text { IRZ } & =\text { inner recirculation zone } \\ \text { ISL } & =\text { inner shear layer } \\ \text { PtF } & =\text { power-to-fuel } \\ \text { ORZ } & =\text { outer recirculation zone } \\ \text { OSL } & =\text { outer shear layer } \\ \text { PVC } & =\text { precessing vortex core } \\ \text { PIV } & =\text { particle image velocimetry } \\ \text { PLIF } & =\text { planar laser-induced fluorescence } \\ \text { dt } & =\text { pulse separation time } \\ \text { Sr } & =\text { Strouhal number } \\ ()_{\text {st }} & =\text { stoichiometric } \\ ()_{\mathrm{i}} & =\text { inner } \\ ()_{0} & =\text { outer }\end{array}$

\section{Introduction}

$\mathrm{A}$

lternative fuels for air and ground transport are increasingly important due to environmental concerns and a finite supply of crude oil based fuels ${ }^{1,2}$. Such fuels can be produced from a variety of sources, including fossil sources such as coal or natural gas, and renewable sources such as biomass. Furthermore, Power-toFuel (PtF) processes are gaining increased interest ${ }^{3}$. Several production pathways exist for alternative fuels, such as Fisher-Tropsch ${ }^{4}$, Direct Alcohol-to-Jet (AtJ) ${ }^{5}$, and many more ${ }^{1}$. These production pathways offer control of the chemical composition of the fuel, thus allowing an optimization of the physical, chemical, and combustion/emission properties. Depending on the feedstock and the processing parameters, alternative fuels can contain hydrocarbons of significantly different types and chain lengths. For a detailed understanding of the combustion behavior of different fuels, knowledge of the combustion properties of the single fuel components is of great importance $e^{6-8}$. However, the influence of the chemical composition of the fuel on combustion performance is not fully understood ${ }^{1}$.

The combustion properties of alternative aviation fuels in gas turbine model combustors have been evaluated in several studies. For example, Sidey et al. studied the effect of methane addition on flame stabilization and local extinction in dual-fuel swirl flames with liquid ethanol and gaseous methane ${ }^{9}$, as well as fuel effects on a kerosene swirl flame with Jet A-1 and $\mathrm{AtJ}^{10}$. Grohmann et al. examined the combustion behavior of single component fuels in a spray gas turbine model combustor in terms of combustion characteristics ${ }^{6}$, fuel effects on the lean blow-out limit ${ }^{7}$, and on pollutant emissions ${ }^{8}$, as well as on the temperature field within the combustion chamber ${ }^{11}$. Similarly, Huelskamp et al. ${ }^{12,13}$ studied fuel effects on the lean blow-out limit in a bluff-body stabilized flame.

In technical applications, the combustion of liquid fuels is governed by four main processes: atomization, vaporization, turbulent mixing and chemical reaction. These processes occur simultaneously, have strong interactions, and cannot easily be decoupled. Prevaporizing liquid fuels has the potential to separate physical and chemical processes, allowing the study of the combustion properties of different fuels based primarily on their chemical properties.

In the current study, a gas turbine model combustor (GTMC) was adapted and set-up for the study of prevaporized single component fuels. Prevaporized ethanol was supplied to the combustor using a direct prevaporizer. All supply lines and the combustion air were preheated to $\mathrm{T}>100^{\circ} \mathrm{C}$ in order to avoid fuel condensation within the supply lines. Optical diagnostics, namely stereoscopic particle image velocimetry (S$\mathrm{PIV}), \mathrm{OH}$ planar laser-induced fluorescence (OH PLIF) and $\mathrm{OH}^{*}$ chemiluminescence imaging $\left(\mathrm{OH}^{*} \mathrm{CL}\right)$, were applied at a sustained repetition rate of $10 \mathrm{kHz}$ to measure the flow-field and flame dynamics at different operating conditions. Additionally, pressure fluctuations within the air plenums and within the combustion chamber were measured using calibrated microphone probes. The goal of the current study is (a) to present this novel setup for studying prevaporized liquid fuel flames in a technically relevant geometry and (b) to gain insight into the flame structure and dynamic processes occurring in such flames. Dominant frequencies for the studied operating conditions were studied by means of fft-analysis of the pressure and heat release rate fluctuations. Furthermore, spatially resolved fft-analysis of the OH PLIF signal allowed identification of regions where specific modes are dominant. 


\section{Experimental}

\section{A. SFB Dual Swirl Burner}

A schematic of the combustor is shown in Figure 1. Details on the geometry can be found in the literature ${ }^{14-}$ ${ }^{16}$, and only a brief overview is provided here. The design is based on previously studied dual-swirlcombustor configurations, but features several improvements to the boundary conditions. Most importantly, the combustor features two swirlers with separate plenum chambers. Thus, the air mass flow to each plenum can be controlled independently, such that the air split ratio $(L)$ between the inner and outer nozzle (defined as the ratio of the air mass flows of the outer $\left(\dot{m}_{\text {air }, o}\right)$ and inner plenum $\left.\left(\dot{m}_{\text {air }, i}\right)\right)$ can be set exactly. Fuel is supplied to the inner nozzle through a feed system built into the splitter wall between the nozzles. The fuel flows through a circle of 60 holes, which are $0.5 \mathrm{~mm}$ in diameter and are located on the inner wall surface $12 \mathrm{~mm}$ below the nozzle exit. In this configuration, the flow from the inner nozzle is partially premixed before the onset of combustion.

The combustion chamber offers very good optical access from all four sides and it is equipped with several ports for pressure probes. A nearly identical setup is available for liquid fuels ${ }^{17}$. Further, the design allows for several identical burners to be combined into a combustor array, in order to investigate cross-flame interactions ${ }^{18}$. Previous studies have focused on the feedback mechanism of the thermo-acoustic cycle ${ }^{14}$, the variations of the thermo-chemical state throughout

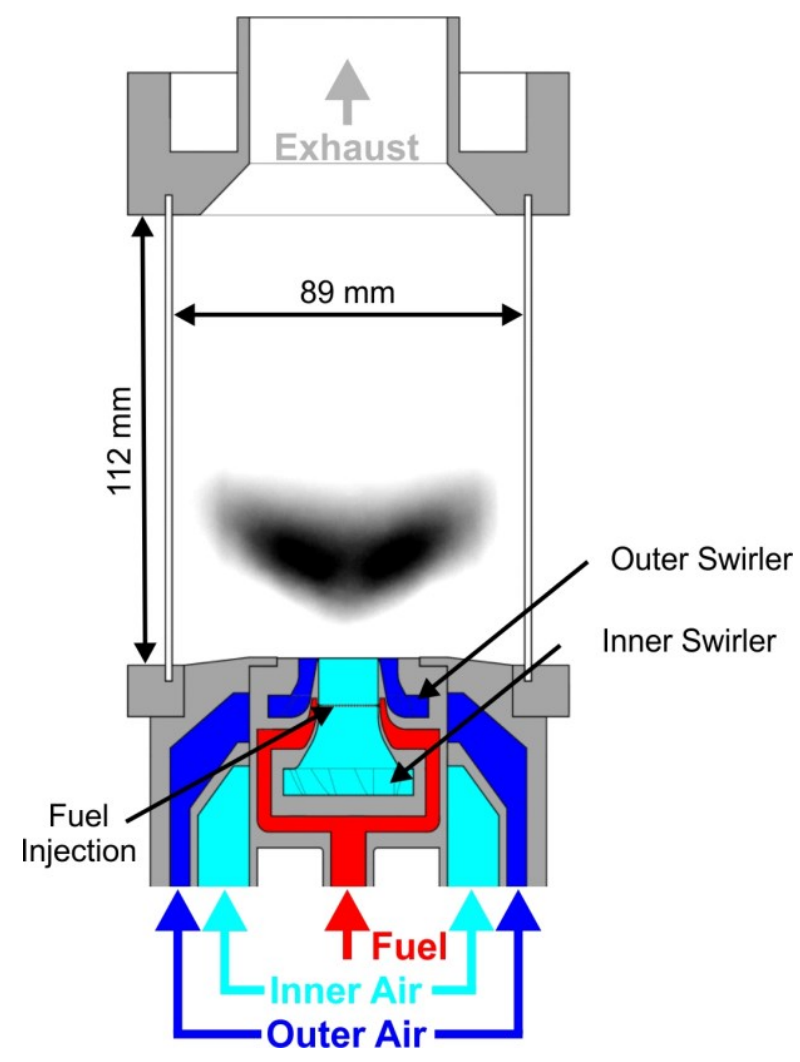

Figure 1. Detail of the nozzle and combustion chamber of the SFB dual-swirl-burner.

the acoustic cycle ${ }^{15}$, the influence of the air split ratio on the dynamics of a precessing vortex core (PVC) ${ }^{16}$ and the impact of the material temperature on thermo-acoustic properties of the burner ${ }^{19}$.

In the current experiments, the combustion dynamics of prevaporized liquid fuel flames (here: ethanol) were studied using high speed optical diagnostics. An overview of the operating conditions studied in this paper is shown in Table 1 .

\begin{tabular}{crrrrr}
\hline$L$ & $\dot{m}_{\text {air }, i}$ & $\dot{m}_{\text {air }, o}$ & $\dot{m}_{C_{2} H_{6} O}$ & $P_{\text {th }}$ \\
{$[-]$} & {$[\mathrm{g} / \mathrm{min}]$} & {$[\mathrm{g} / \mathrm{min}]$} & {$[\mathrm{g} / \mathrm{min}]$} & $\begin{array}{c}\varphi_{\text {global }} \\
{[\mathrm{kW}]}\end{array}$ & $\begin{array}{c}\text { [-] } \\
{[1.6}\end{array}$ \\
1.6 & 154 & 246 & 24.4 & 10.91 & 0.55 \\
1.6 & 154 & 246 & 28.9 & 12.89 & 0.65 \\
1.6 & 154 & 246 & 33.3 & 14.88 & 0.75 \\
\hline
\end{tabular}

Tab. 1 Operating conditions for the combustor. $L$ is the air split ratio between the outer and the innter swirler. $\dot{m}_{a i r, i}$ and $\dot{m}_{a i r, o}$ air the air mass flows for the inner and outer swirler, $\dot{m}_{C_{2} \mathrm{H}_{6} \mathrm{O}}$ is the mass flow of ethanol. $P_{t h}$ is the global thermal power and $\varphi_{\text {global }}$ is the global equivalence ratio.

The media supply to the burner and the flow instrumentation are shown in Figure 2. Fuel was supplied to a direct prevaporizer (aSTEAM DV1) using a mass flow controller (Bronkhorst Mini Coriflow) fed by a piston accumulator, which was pressurized using nitrogen. All fuel lines were heated to a temperature of $T_{\text {fuel }}=150^{\circ} \mathrm{C}$ to avoid condensation in the fuel supply. Additionally, the air was preheated to $T_{\text {air }}=100^{\circ} \mathrm{C}$ using two electrical air heaters (Osram Sylvania Threaded Inline) to avoid condensation in the nozzle. The air and fuel temperatures were monitored using thermocouples close to the air swirlers and inside the fuel plenum, respectively. 
B. High-Speed OH Planar LaserInduced Fluorescence

A schematic of the experimental setup is shown in Figure 3. OH planar laserinduced fluorescence (PLIF) was measured at a frame rate of $10 \mathrm{kHz}$ using a Sirah Credo Dye laser pumped by an diode-pumped solid state (DPSS) laser (Edgewave Innoslab IS400-2-L). The frequency doubled output of the dye laser was tuned to $283.2 \mathrm{~nm}$ to match the $\mathrm{Q}_{1}(7)$ transition of $\mathrm{OH}$ in the $\mathrm{A}-\mathrm{X}\left(v^{\prime \prime}=1, v^{\prime}=\right.$ 0 ) band. The laser beam was expanded into a light sheet using a two-stage cylindrical telescope and focused into the test section using a third cylindrical lens, resulting in a laser sheet of approximately $45 \mathrm{~mm}$ height with a beam waist in the test section of $0.4 \mathrm{~mm}$. The fluorescence signal in the $(1,1)$ and $(1,0)$ band around $308 \mathrm{~nm}$ was collected using a high speed CMOS camera (Photron SA5) equipped with a two-stage high speed image intensifier (LaVision HS IRO), a Halle $f=64 \mathrm{~mm}$ f/2 UV lens and a high transmission ( $\mathrm{T}>80 \%$ at $310 \mathrm{~nm}$ ) band pass filter. A part of the light sheet was directed into a dye cell containing Rhodamine 6G solved in ethanol to correct for shot-to-shot sheet profile and laser energy fluctuations. Fluorescence from the dye cell was imaged using a Photron SA1.1 high-speed CMOS camera equipped with a Nikon Nikkor $f=50 \mathrm{~mm}$ f/1.4 lens.

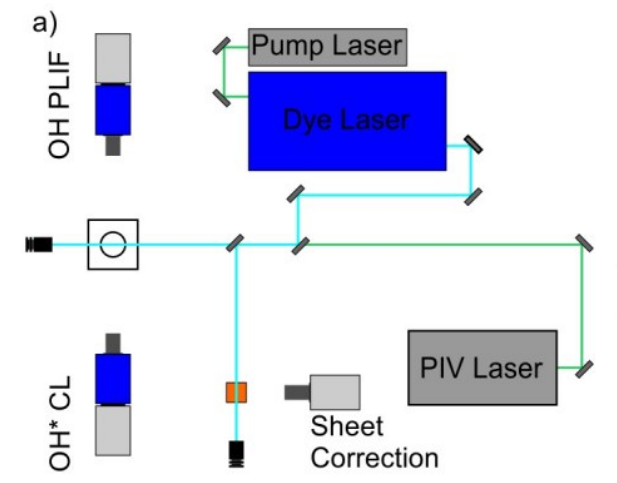

b)

Figure 3. Experimental Setup. a) top view b) front view. 


\section{High Speed Stereoscopic Particle Image Velocimetry (HS S-PIV)}

The three-component velocity field in the measurement plane was acquired with Stereoscopic Particle Image Velocimetry (S-PIV) at a repetition rate of $10 \mathrm{kHz}$. Titanium dioxide $\left(\mathrm{TiO}_{2}\right)$ particles were added to the flow and illuminated with a frequency doubled dual-head DPSS Nd:YAG laser (Edgewave Innoslab IS200-2-LD) with a pulse energy of $5 \mathrm{~mJ}$ at $532 \mathrm{~nm}$ and a pulse separation time $d t$ between $15 \mu \mathrm{s}$ and $20 \mu \mathrm{s}$, depending on the operating condition. Mie scattering from the particles was recorded with a pair of high speed CMOS cameras (Phantom V1212). The cameras were mounted at an angle of $30^{\circ}$ relative to the laser light sheet and equipped with Tokina $f=100 \mathrm{~mm}$ macro lenses (set to $\mathrm{f} / 5.6)$ and band-pass filters $(532 \pm 5 \mathrm{~nm}$ ) to suppress background luminosity of the flame. A total of 10,000 single shot image pairs were recorded for each operating condition and the velocity fields were calculated from the particle image pairs using a multi-scale cross-correlation PIV algorithm (LaVision DaVis 8.4) with a final interrogation window size of $24 \times 24$ pixels with $50 \%$ overlap. The resulting spatial resolution and vector spacing were $1.3 \mathrm{~mm}$ and $0.65 \mathrm{~mm}$, respectively.

\section{D. $\mathrm{OH}^{*}$ Chemiluminescence (CL)}

$\mathrm{OH}^{*}$ chemiluminescence was measured simultaneously with the OH PLIF and S-PIV measurements using a Photron SA5 high speed CMOS camera equipped with a LaVision HS IRO image intensifier. The intensifier was equipped with a Cerco $f=45 \mathrm{~mm} \mathrm{f} / 1.8 \mathrm{UV}$ lens and a bandpass filter centered around $310 \mathrm{~nm}$ to collect $\mathrm{OH}^{*} \mathrm{CL}$ in the $(1,0)$ and $(1,1)$ band.

\section{E. Acoustic Measurements}

Pressure oscillations in the combustion chamber and in the two air plenums were measured using calibrated microphone probes (Brüel \& Kjær, type 4939), with a sampling rate of $100 \mathrm{kHz}$. The microphone probes were calibrated for frequencies up to $10 \mathrm{kHz}$. The pressure power spectrum at each location was computed by slicing the long-duration pressure signal into one-second segments, and calculating the power spectrum for each segment. Afterwards, the spectra of the segments were averaged, resulting in a frequency resolution of $1 \mathrm{~Hz}$. No additional filtering or smoothing of the raw signals or the frequency spectra was performed. Acoustic modes leading to an asymmetric pressure distribution in the combustion chamber, such as transversal thermo-acoustic modes or asymmetric flow instabilities like precessing vortex cores (PVCs), could be detected by calculating the frequency spectrum of the difference signal of two microphone probes that were mounted on opposite sides of the combustion chamber, but at the same axial position. 


\section{Results and Discussion}

An overview of the different flame shapes for the studied operating conditions is given by the mean and standard deviation of the $\mathrm{OH}^{*} \mathrm{CL}$ signal, given in Figure 4.
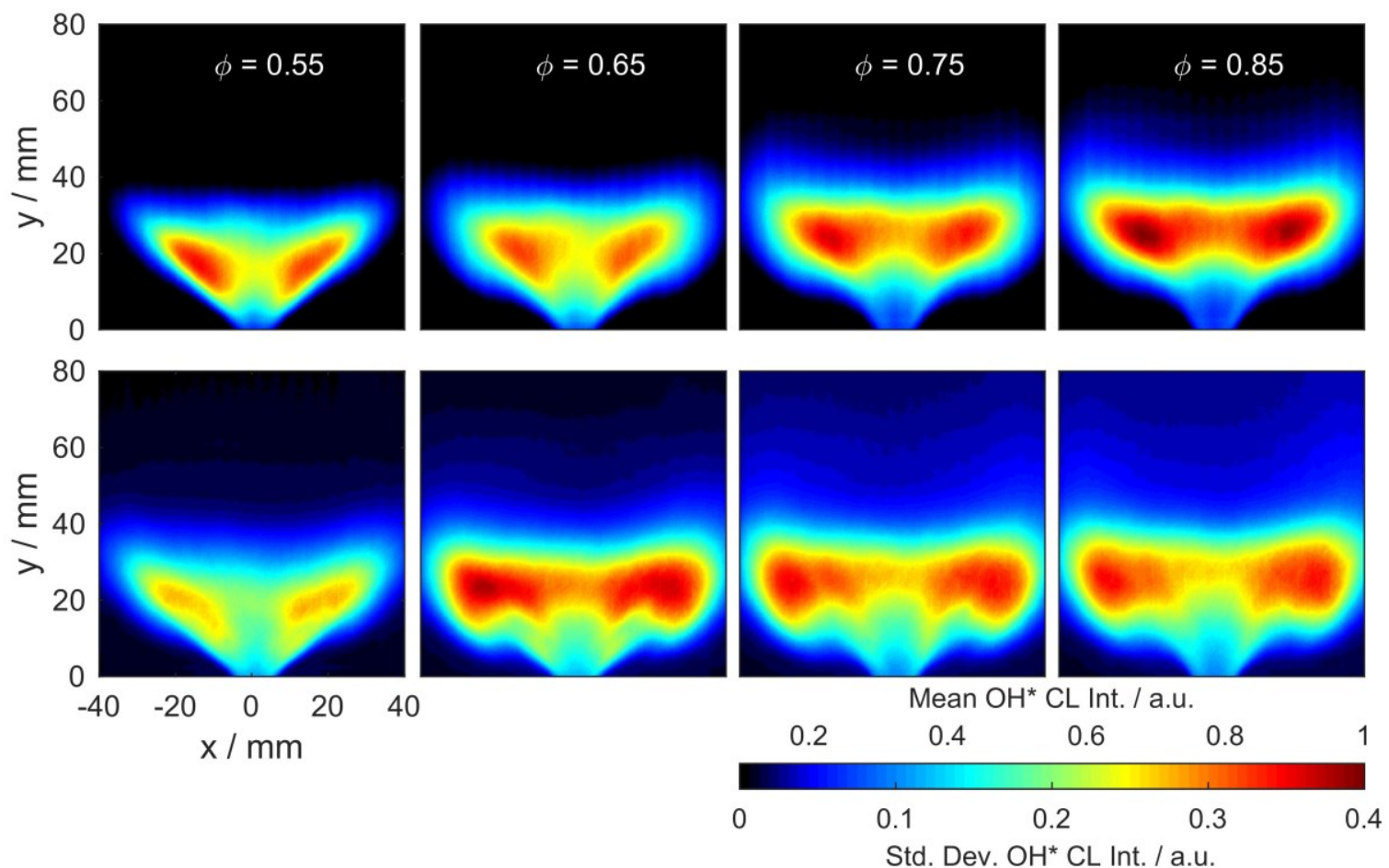

Figure 4. Mean (top row) and standard deviation (bottom row) $\mathrm{OH}^{*}$ chemiluminescence distribution for the different studied operating conditions.

The top row corresponds to the mean $\mathrm{OH}^{*} \mathrm{CL}$ signal, and the bottom row corresponds to the standard deviation. The equivalence ratio $\varphi$ increases from left to right. All flames exhibited a "V"-shape; the flame root is inside the nozzle for all operating conditions. With increasing equivalence ratio (and thus increasing thermal power), the overall $\mathrm{OH}^{*} \mathrm{CL}$ intensity increases. The main flame zone (visualized by the maximum of the $\mathrm{OH}^{*} \mathrm{CL}$ signal) is shifted further downstream with increasing $\varphi$, while the axial extent of the main flame zone remains similar. For the lower equivalence ratios $\varphi=0.55$ and $\varphi=0.65$, the flame is attached. For increasing equivalence ratio $(\varphi=0.75$ and $\varphi=0.85)$ the main flame zone appears to be lifted from the fuel nozzle, and only low $\mathrm{OH}^{*} \mathrm{CL}$ signal intensities are visible at the flame root. A similar trend is observed for the standard deviation. Since all examined flames feature self-excited thermo-acoustic oscillations (as will be discussed below) that are coupled with heat release fluctuations, the variances in the $\mathrm{OH}^{*} \mathrm{CL}$ signal intensity mainly stem from those oscillations. With increasing equivalence ratio, the area of high $\mathrm{OH}^{*} \mathrm{CL}$ fluctuations is shifted further downstream. Furthermore, increased fluctuations can be observed in the outer recirculation zone (ORZ) with increasing $\varphi$. However, for all flame cases, significant $\mathrm{OH}^{*} \mathrm{CL}$ fluctuations are visible close to the nozzle, which will be explained below.

An overview of the thermo-acoustic properties of the examined flames is given in Figure 5, where, the frequency spectra within the combustion chamber, the inner plenum and outer plenum are shown. For all examined operating conditions, the dominant thermo-acoustic mode is at $460 \mathrm{~Hz}$ and, in contrast to flames with methane as fuel and non-preheated air $^{14,16}$, no shift in the dominant frequency with increasing equivalence ratio (and coupled the thermal power) is observed. In correspondence with a previous study ${ }^{14}$, taking into account the different acoustic velocities for cold and preheated air, this mode is close to a resonance mode with the length of the inner plenum $\left(\lambda=2 \times 1_{I P}\right)$, where $1_{I P}$ corresponds to the length of the inner plenum $(380 \mathrm{~mm})$.

An additional mode at approximately $570 \mathrm{~Hz}$ is observed and increases in strength with increasing equivalence ratio. While at $\varphi=0.85$, this mode shows a similar amplitude as the mode at $460 \mathrm{~Hz}$, it is not observed for the leanest studied operating condition $(\varphi=0.55)$. The second harmonic of $460 \mathrm{~Hz}$ mode at $920 \mathrm{~Hz}$ is most dominant at $\varphi=0.65$ and $\varphi=0.75$. 

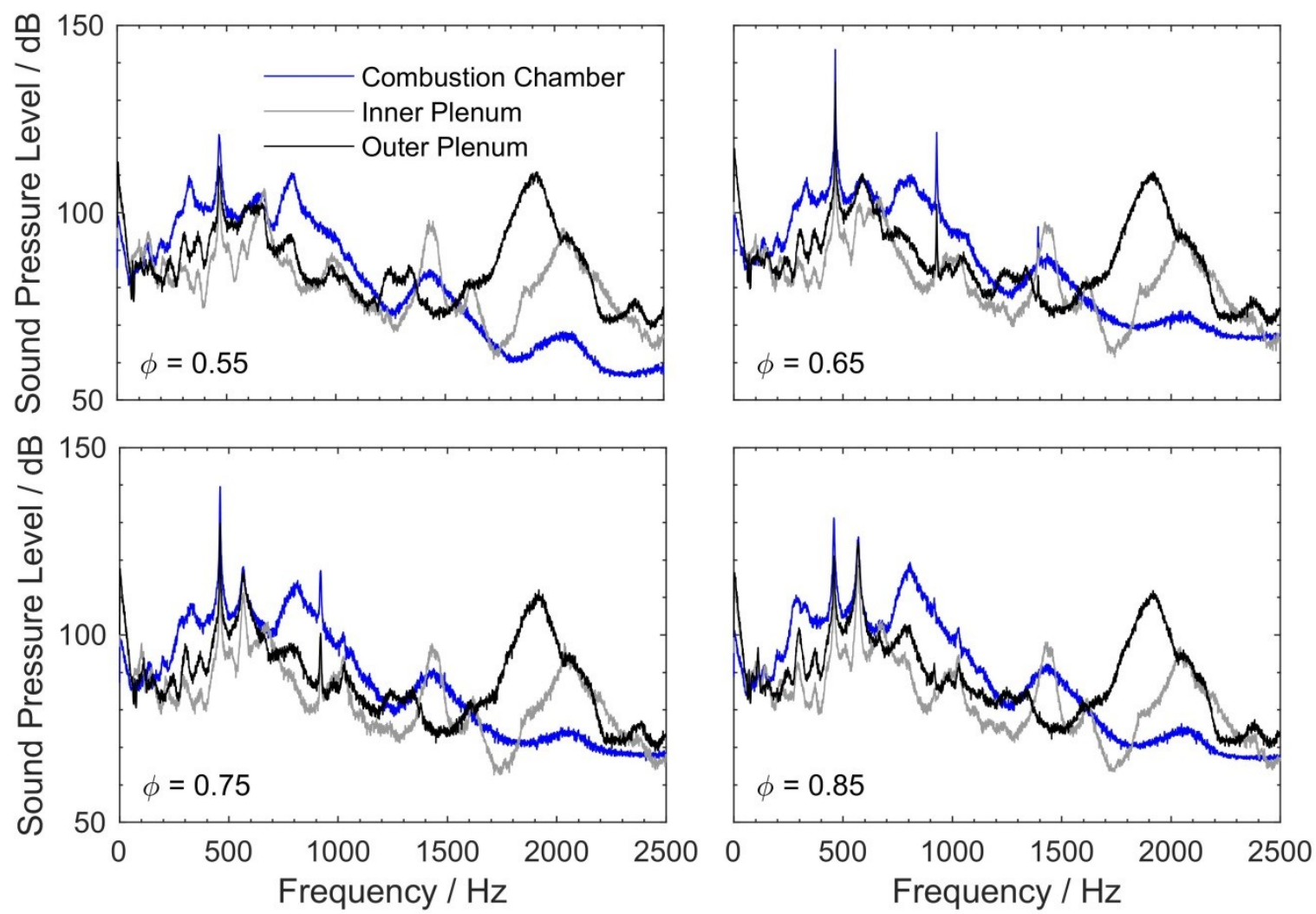

Figure 5 Acoustic spectra from the combustion chamber, and inner and outer plenum for the examined operating conditions.

Additional information on the influence of the observed acoustic modes on the flame dynamics can be gained via a frequency analysis of the $\mathrm{OH}^{*} \mathrm{CL}$ signal. Here, a fast fourier transform (fft) was performed for each pixel of the $\mathrm{OH}^{*} \mathrm{CL}$ images, and the corresponding ffts were averaged to gain one single frequency spectrum per operating condition. The corresponding frequency spectra are shown in Figure 6.

For all operating conditions, the dominant mode in the $\mathrm{OH}^{*} \mathrm{CL}$ signal is at approximately $460 \mathrm{~Hz}$, and the corresponding second harmonic mode occurs at approximately $930 \mathrm{~Hz}$ for most operating conditions. The dominant thermo-acoustic mode for the here presented operating conditions with preheated air and prevaporized ethanol as fuel is similar to the dominant thermo-acoustic frequency for methane flames with air at room temperature, which was $392 \mathrm{~Hz}^{14}$. Further modes visible in the $\mathrm{OH}^{*} \mathrm{CL}$ frequency spectrum occur at $115 \mathrm{~Hz}$ (which is close to the Helmholtz resonance mode of $86 \mathrm{~Hz}$ for the inner plenum for methane flames with air at room temperature ${ }^{14}$ ), $575 \mathrm{~Hz}$ (which is in the range of observed frequencies for a precessing vortex core for the current combustor $^{14,16}$ ) and $1400 \mathrm{~Hz}$.

The frequency peak at $460 \mathrm{~Hz}$ shows the

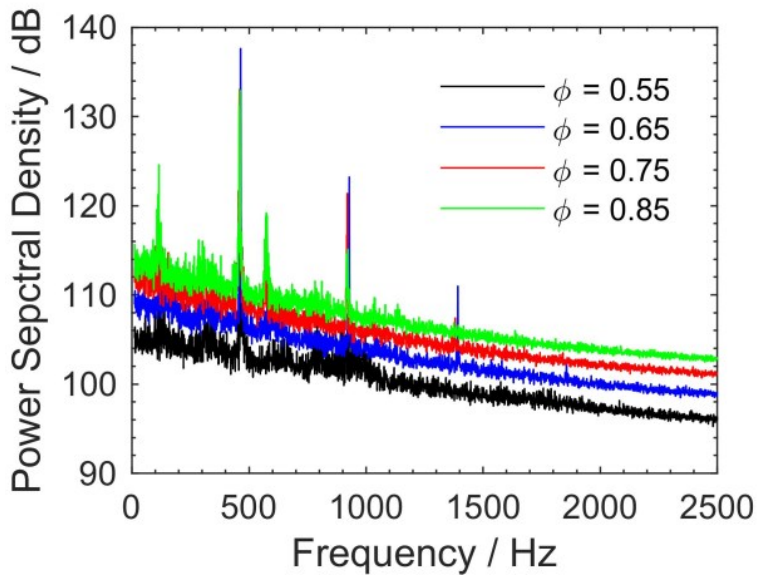

Figure 6 Frequency spectra of the OH* CL signal for the examined operating conditions

highest amplitude for $\varphi=0.65$, while it is almost not visible for $\varphi=0.55$. For $\varphi=0.75$ and $\varphi=0.85$, the amplitude of the frequency peak at $460 \mathrm{~Hz}$ is comparable. Similarly, the second harmonic of the dominant frequency at $930 \mathrm{~Hz}$ exhibits the highest amplitude for $\varphi=0.65$. In contrast, the modes at $115 \mathrm{~Hz}$ and $575 \mathrm{~Hz}$ exhibit the highest amplitudes for $\varphi=0.85$.

A more detailed insight of the flame dynamics can be gained by a fft analysis of the OH PLIF signal intensity, which, in contrast to the line-of-sight integrated $\mathrm{OH}^{*} \mathrm{CL}$ measurements, yields information with high spatial resolution due to the $2 \mathrm{D}$ nature of the PLIF measurements. To gain an initial overview of the $\mathrm{OH}$ 
distribution within the test section, Figure 7 shows the mean and standard deviation of the OH PLIF signal for the studied operating conditions.
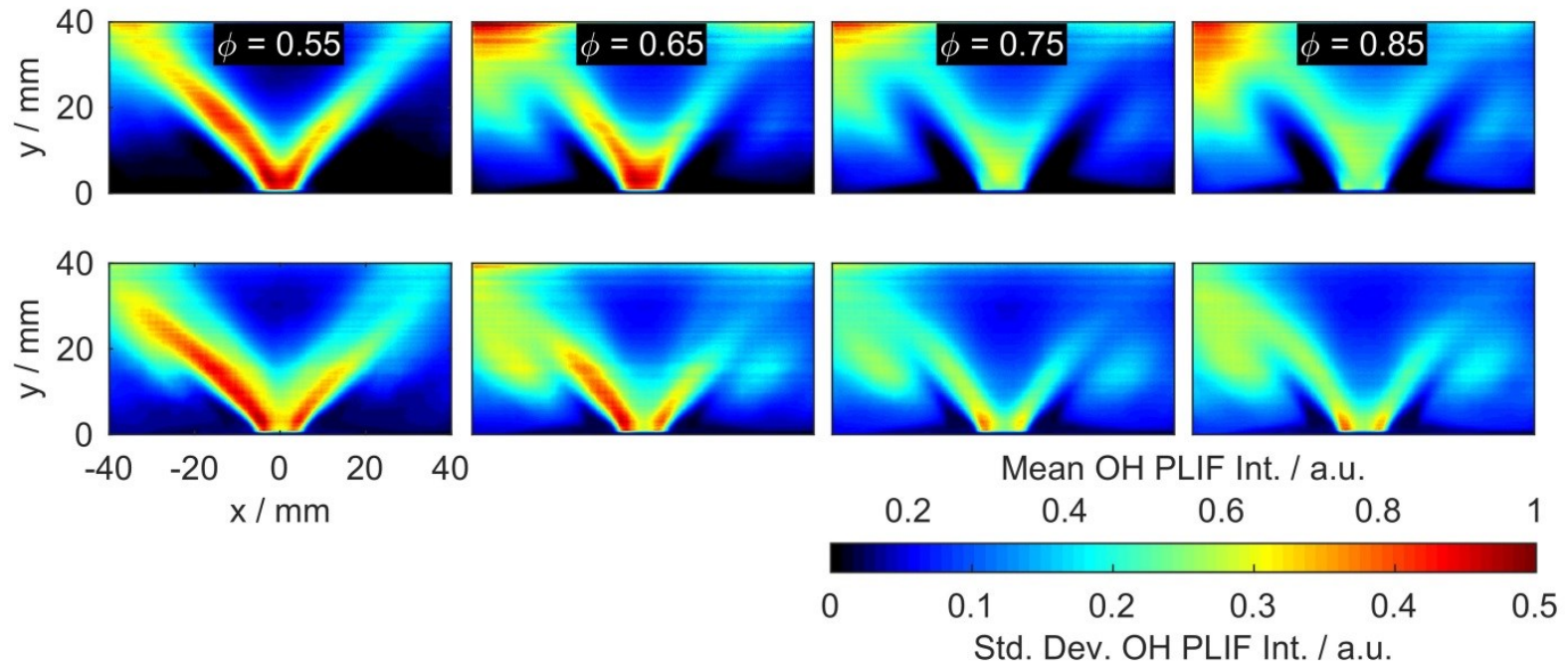

Figure 7. Mean (top row) and standard deviation (bottom row) of the OH PLIF signal for the examined operating conditions.

The top row corresponds to the mean OH PLIF signal, and the bottom row corresponds to the standard deviation. The asymmetry observed for all operating conditions (higher signal intensities at the left-hand side of the images) is due to laser absorption by $\mathrm{OH}$ in the test section and lower fluorescence intensity with lower local laser energy further away from the laser entry side of the combustion chamber. For $\varphi=0.55$, the maximum $\mathrm{OH}$ PLIF signal is close to the fuel nozzle and extends along the inner shear layer, with little $\mathrm{OH}$ being present in the inner and outer recirculation zones. With increasing equivalence ratio, an increased OH PLIF signal in the outer recirculation zone (ORZ) is observed. Within the inner shear layer (ISL), the $\mathrm{OH}$ distribution becomes less homogeneous with increasing equivalence ratio. With increasing $\varphi$, higher signal intensities are observed for $y>20 \mathrm{~mm}$, which is in agreement with the observed shift in maximum $\mathrm{OH}^{*} \mathrm{CL}$ signal intensity with increasing $\varphi$ (cf. Figure 4). For higher equivalence ratios, the maximum OH PLIF signal within the ISL occurs close to the nozzle and gradually decreases with increasing axial distance from the nozzle. The observed trend of increasing OH PLIF signal in the ORZ with increasing equivalence ratio (and thus increasing thermal power) is probably due to higher gas temperatures and lower thermal losses at the combustion chamber windows. Additionally, the $\mathrm{OH}$ PLIF signal in the inner recirculation zone increases with increasing $\varphi$, and for $\varphi=0.85$, significant $\mathrm{OH}$ concentrations are present in the inner and outer recirculation zone.

The standard deviation of the OH PLIF signal shows a similar behavior as the mean signal. For $\varphi=0.55$, the strongest fluctuations occur in the ISL, but almost no fluctuations are visible in the OSL and ORZ. With increasing equivalence ratio, the fluctuations in the outer shear layer (OSL) and ORZ increase, and are maximal for $\varphi=0.85$. For all cases, strong fluctuations are visible directly downstream of the nozzle, as will also be discussed below based on time series of single shots.

Figure 8 shows the flow field for the different operating conditions. Color coded are the mean (top row) and standard deviation (bottom row) of the axial velocity component. The overlay corresponds to the stream lines. 

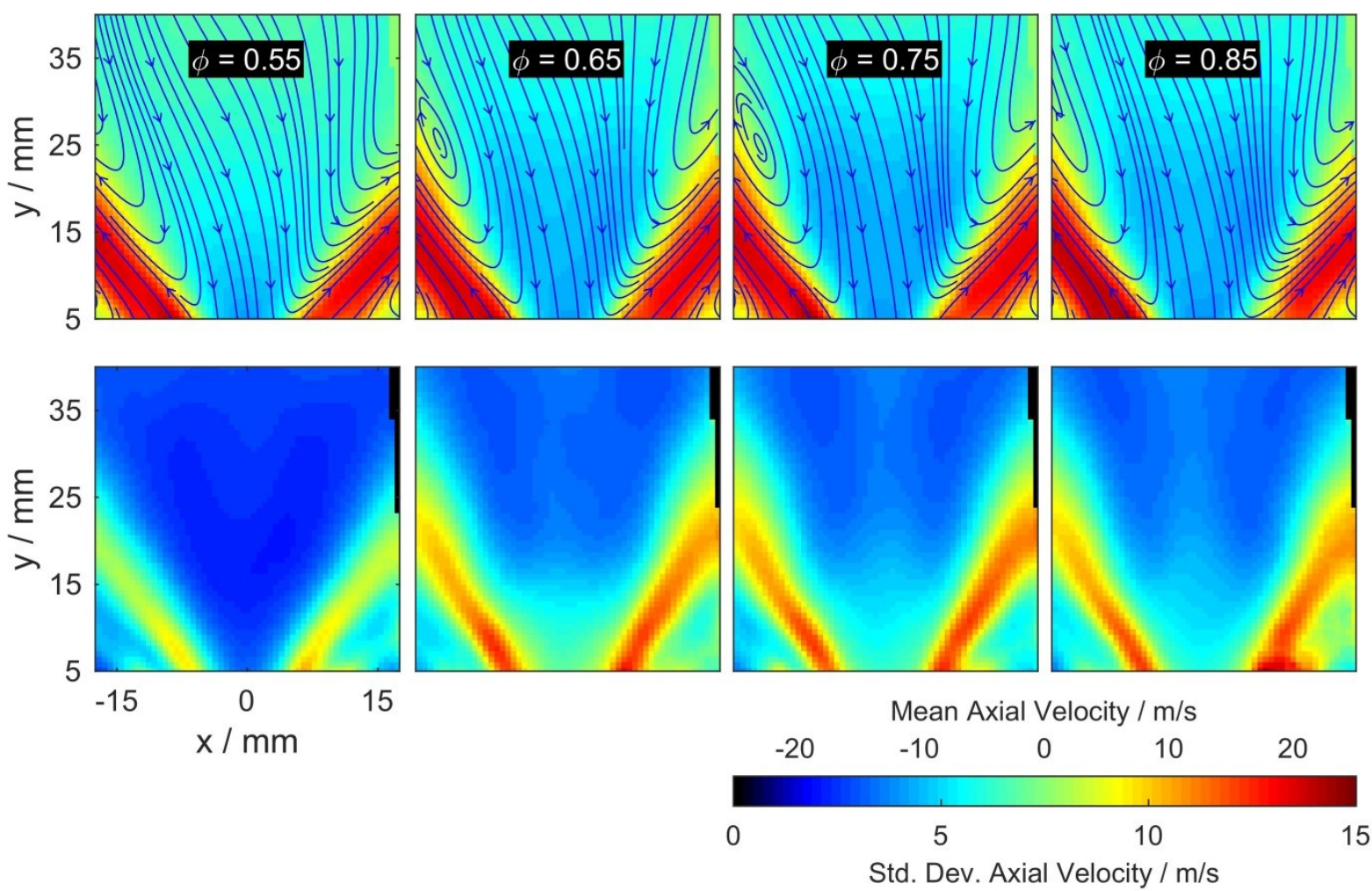

Figure 8. Mean (top row) and standard deviation (bottom row) of the axial velocity component for the studied operating conditions. For the mean flow fields, the stream lines are shown as well

Since the air mass flow is constant for all operating conditions, the mean flow field is expected to be similar for all operating conditions. Indeed, the maximum velocity magnitude in the inflow region only increases slightly with increasing equivalence ratio (and thus thermal power and adiabatic flame temperature). The shape of the flow field and position of the recirculation bubble is similar for all operating conditions. The standard deviation of the axial velocity component, however, shows significant difference between the operating conditions. For $\varphi=0.55$, the lowest velocity fluctuations occur, with almost no fluctuations in the inner recirculation zone. With increasing equivalence ratio, the velocity fluctuations in the inflow region and in the inner recirculation zone increase significantly. As expected, the maximum velocity fluctuations are found in the inner and outer shear layers surrounding the inflow region. Large velocity fluctuations are also visible close to the flame root. The coupling of the velocity fluctuations with the flame zone can be evaluated by identifying regions in which certain frequency modes are dominant in the OH PLIF signal.

The frequency-dependent dynamics of the OH PLIF signal are visualized in Figure 9.
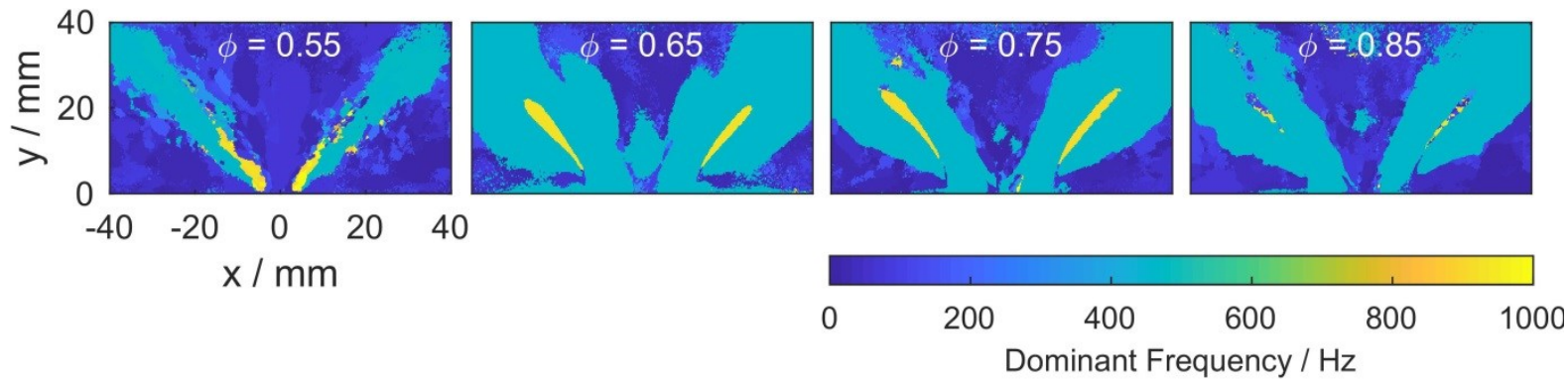

Figure 9. Spatial distribution of dominant frequencies of the OH PLIF signal. The green regions correspond to the most dominant mode at $460 \mathrm{~Hz}$, the yellow regions to the second harmonic of this mode at $920 \mathrm{~Hz}$ and the dark blue regions to a frequency of approximately $75 \mathrm{~Hz}$.

Here, a frequency spectrum was computed for the time series of the OH PLIF signal for each camera pixel, and the dominant frequency was extracted. Color coded is the frequency of the most dominant mode of the frequency spectrum for each measurement location. The cyan/green colors correspond to a frequency of $460 \mathrm{~Hz}$, which was already identified as the dominant mode in the $\mathrm{OH}^{*} \mathrm{CL}$ signal. The yellow areas correspond to the second harmonic peak of the $460 \mathrm{~Hz}$ mode (i.e. frequencies of approximately $920 \mathrm{~Hz}$ ). In those areas, the second harmonic of the overall dominant mode is stronger as the first harmonic. The dark blue regions, which are 
mainly visible in the IRZ for the $\varphi=0.55$ case correspond to frequencies of approximately $75 \mathrm{~Hz}$. Modes corresponding to this frequency were not identified in the $\mathrm{OH}^{*} \mathrm{CL}$ measurements, However, the frequency of this mode is approximately half of the Helmholtz mode at $150 \mathrm{~Hz}$, which was observed in the $\mathrm{OH}^{*} \mathrm{CL}$ measurements.

With increasing equivalence ratio (and coupled increasing thermal power), fluctuations corresponding to the dominant mode at $460 \mathrm{~Hz}$ are increasing in the OSL (and also sometimes in the IRZ close to the nozzle). Apart from this area, few oscillations at the dominant mode are visible in the IRZ and ORZ.

Figure 10 shows temporal sequences of $\mathrm{OH}$ PLIF, demonstrating the different flame dynamics for the different operating conditions.
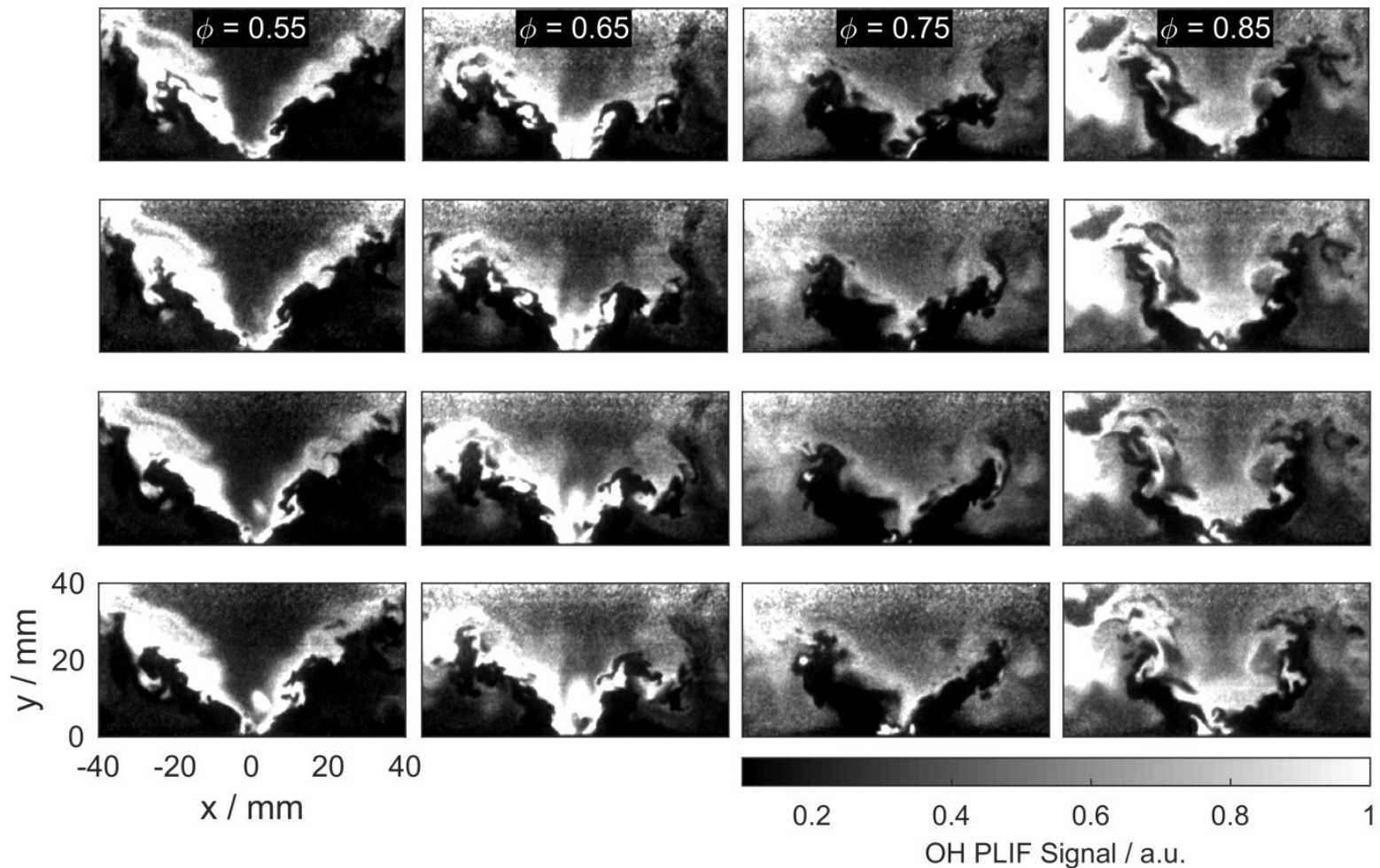

Figure 10. Temporal dynamics of the OH PLIF signal for the different studied operating conditions. The time step between each image row is $0.1 \mathrm{~ms}$.

The time between frames is $0.1 \mathrm{~ms}$, corresponding to a frame rate of $10 \mathrm{kHz}$. All operating conditions feature vortex shedding and flame roll-up due to vortices is frequently observed. For example, for $\varphi=0.65$ and $\varphi=0.75$, a flame roll-up due to vortex shedding can be observed on the right-hand side of the test section. In regions where vortex shedding occurs, high OH PLIF intensities are observed, for example for $\varphi=0.55$, where slight vortex shedding can be observed at the left-hand side of the test section, followed by an increase OH PLIF signal intensity in the shear layer. A comparable behavior was observed in swirl flames with a similar geometry ${ }^{20,21}$. For $\varphi=0.85$, a flame segment can be seen forming in the center of a vortex on the right-hand side of the test section. This could either be due to auto-ignition in the center of the vortex core, where long residence times and low strain rates are expected, or due to transport of a flame segment from outside the measurement plane $e^{22,23}$. Albeit similar fluid-dynamic effects are observed for all operating conditions, the overall structure of the $\mathrm{OH}$ layer changes with varying operating condition. For $\varphi=0.55$ (left column), a large zone of super-equilibrium $\mathrm{OH}$ (visualized by the broad area of increased $\mathrm{OH}$ signal inside the inflow) can be seen, while almost no $\mathrm{OH}$ is visible in the inner recirculation zone (IRZ). This changes when increasing the equivalence ratio to $\varphi=0.65$ (second column from the left). Here, an increased OH PLIF signal can be seen in the IRZ, indicating strong recirculation of hot, $\mathrm{OH}$-containing exhaust gas. Increasing the equivalence ratio further to $\varphi=0.75$ (second column from the right) leads to further dynamic processes. Here, local extinction is visible close to the flame root. For $\varphi=0.85$, almost no flame reactions can be observed at the flame base, except for some "pilot flame" areas inside and slightly above the burner nozzle, which seem to play a key role in the flame stabilization mechanism under those boundary conditions. The high velocity fluctuations at the flame root (cf. Figure 8) could lead to the here observed local extinction. Since the dominant frequency of the OH PLIF signal at locations where local extinction is observed (cf. Figure 9) is at the frequency of the dominant thermo-acoustic mode, the observed local extinction seems to be coupled to the thermo-acoustic oscillations. The increased occurrence of 
local extinction at the flame root with increasing equivalence ratio is also a possible explanation for the observed downstream shift of the main flame zone (cf. Figure 4) with increasing equivalence ratio.

\section{Conclusions and Outlook}

A dual swirl gas turbine model combustor was setup for pre-vaporized liquid fuel flames and studied using high-speed laser diagnostics. The flame exhibited a V-shape for all operating conditions. While the flame root was inside the burner nozzle, the main reaction zone was lifted, and shifted further downstream with increasing equivalence ratio. All flames exhibited thermo-acoustic oscillations at a frequency of approximately $460 \mathrm{~Hz}$, with a general trend of increasing sound pressure level with increasing equivalence ratio. The same mode was also identified in a frequency analysis of the $\mathrm{OH}^{*}$ chemiluminescence and $\mathrm{OH}$ PLIF signals. Several additional modes were identified in the acoustic and $\mathrm{OH}^{*} \mathrm{CL}$ signals.

In comparison to cases with gaseous methane as fuel and non-preheated air in the same combustor geometry, a different behavior in terms of thermo-acoustics was observed. While for the methane flames, the dominant frequency of the thermo-acoustic mode increased with increasing equivalence ratio, the dominant frequency remained almost constant for the prevaporized ethanol case. Spatially resolved evaluation of the dominant frequencies within the combustor revealed that the main feedback zone for the thermo-acoustic oscillations is within the inner and outer shear layers.

The studied flames furthermore showed strong fluid-dynamic processes, such as flame-roll up due to vortex shedding. For the higher equivalence ratios studied here, local extinction at the flame root was observed and the increase of lift-off height of the main reaction zone with increasing equivalence ratio was attributed to this effect. Small flame parcels within the nozzle were found to assist the flame stabilization for operating conditions were localized extinction occurs at the flame root. Since the local extinction events occur at the same frequency as the dominant thermo-acoustic mode, these effects seem to be coupled.

\section{Acknowledgements}

Jasper Grohmann and Yawei Gao are gratefully acknowledged for their support in setting up the experiment.

\section{References}

${ }^{1}$ Blakey, S., Rye, L., and Wilson, C. W., "Aviation gas turbine alternative fuels: A review," Proceedings of the Combustion Institute, Vol. 33, No. 2, 2011, pp. 2863-2885.

${ }^{2}$ Kallio, P., Pásztor, A., Akhtar, M. K., and Jones, P. R., "Renewable jet fuel," Current Opinion in Biotechnology, Vol. 26, 2014, pp. 50-55.

${ }^{3}$ Schemme, S., Samsun, R. C., Peters, R., and Stolten, D., "Power-to-fuel as a key to sustainable transport systems - An analysis of diesel fuels produced from CO2 and renewable electricity," Fuel, Vol. 205, 2017, pp. 198-221.

${ }^{4}$ de Klerk, A. "Chapter 10 - Aviation Turbine Fuels Through the Fischer-Tropsch Process," Biofuels for Aviation. Academic Press, 2016, pp. 241-259.

${ }^{5}$ Brooks, K. P., Snowden-Swan, L. J., Jones, S. B., Butcher, M. G., Lee, G. S. J., Anderson, D. M., Frye, J. G., Holladay, J. E., Owen, J., Harmon, L., Burton, F., Palou-Rivera, I., Plaza, J., Handler, R., and Shonnard, D. "Chapter 6 - Low-Carbon Aviation Fuel Through the Alcohol to Jet Pathway," Biofuels for Aviation. Academic Press, 2016, pp. 109-150.

${ }^{6}$ Grohmann, J., O’Loughlin, W., Meier, W., and Aigner, M. "Comparison of the Combustion Characteristics of Liquid Single-Component Fuels in a Gas Turbine Model Combustor," Proceedings of the ASME Turbo Expo. Vol. GT2017-56177, 2016.

${ }^{7}$ Grohmann, J., Rauch, B., Kathrotia, T., Meier, W., and Aigner, M., "Influence of Single-Component Fuels on Gas-Turbine Model Combustor Lean Blowout," Journal of Propulsion and Power, Vol. 34, No. 1, 2017, pp. 97-107.

${ }^{8}$ Grohmann, J., Meier, W., and Aigner, M. "Gas Turbine Model Combustor Emissions of Liquid SingleComponent Fuels," Proceedings of the ASME Turbo Expo. Vol. GT2017-63182, 2017.

${ }^{9}$ Sidey, J., and Mastorakos, E., "Visualisation of turbulent swirling dual-fuel flames," Proceedings of the Combustion Institute, Vol. 36, No. 2, 2017, pp. 1721-1727.

${ }^{10}$ Sidey, J. A., Allison, P. M., and Mastorakos, E. "The effect of fuel composition on swirling kerosene flames," 55th AIAA Aerospace Sciences Meeting. American Institute of Aeronautics and Astronautics, 2017.

${ }^{11}$ Cantu, L. M. L., Grohmann, J., Meier, W., and Aigner, M., "Temperature measurements in confined swirling spray flames by vibrational coherent anti-stokes Raman spectroscopy," Experimental Thermal and Fluid Science, Vol. 95, 2018, pp. 52-59. 
${ }^{12}$ Huelskamp, B., Monfort, J., Kiel, B. V., Neuroth, C., and Caswell, A. W. "Comparison of Alternative Jet Fuel Lean Blowouts for Bluff-Body Stabilized Flames," 54th AIAA Aerospace Sciences Meeting. American Institute of Aeronautics and Astronautics, 2016.

${ }^{13}$ Huelskamp, B. C., Kiel, B. V., and Gokulakrishnan, P., "Influence of Fuel Characteristics in a Correlation to Predict Lean Blowout of Bluff-Body Stabilized Flames," No. 56697, 2015, p. V04BT04A031.

${ }^{14}$ Arndt, C. M., Severin, M., Dem, C., Stohr, M., Steinberg, A. M., and Meier, W., "Experimental analysis of thermo-acoustic instabilities in a generic gas turbine combustor by phase-correlated PIV, chemiluminescence, and laser Raman scattering measurements," Experiments in Fluids, Vol. 56, No. 4, 2015 , p. 69.

${ }^{15}$ Meier, W., Dem, C., and Arndt, C. M., "Mixing and reaction progress in a confined swirl flame undergoing thermo-acoustic oscillations studied with laser Raman scattering," Experimental Thermal and Fluid Science, Vol. 73, 2015, pp. 71-78.

${ }^{16}$ Arndt, C. M., Stöhr, M., Severin, M. J., Dem, C., and Meier, W. "Influence of air staging on the dynamics of a precessing vortex core in a dual swirl gas turbine model combustor," 53rd AIAA/SAE/ASEE Joint Propulsion Conference. American Institute of Aeronautics and Astronautics, 2017.

${ }^{17}$ Bärow, E., Koch, R., and Bauer, H.-J. "Comparison of oscillation modes of spray and gaseous flames," Proceedings of the Eighth Mediterranean Combustion Symposium. 2013, EGTSC-26.

${ }^{18}$ Kraus, C., Harth, S., and Bockhorn, H., "Experimental investigation of combustion instabilities in lean swirlstabilized partially-premixed flames in single- and multiple-burner setup," International Journal of Spray and Combustion Dynamics, Vol. 8, No. 1, 2016, pp. 4-26.

${ }^{19}$ Kraus, C., Selle, L., Poinsot, T., Arndt, C. M., and Bockhorn, H., "Influence of Heat Transfer and Material Temperature on Combustion Instabilities in a Swirl Burner," Journal of Engineering for Gas Turbines and Power, Vol. 139, No. 5, 2016, p. 051503.

${ }^{20}$ Stöhr, M., Arndt, C. M., and Meier, W., "Transient effects of fuel-air mixing in a partially-premixed turbulent swirl flame," Proceedings of the Combustion Institute, Vol. 35, No. 3, 2015, pp. 3327-3335.

${ }^{21}$ Stöhr, M., Arndt, C. M., and Meier, W., "Effects of Damkohler number on vortex-flame interaction in a gas turbine model combustor," Proceedings of the Combustion Institute, Vol. 34, No. 2, 2013, pp. 3107 3115 .

${ }^{22}$ Boxx, I., Arndt, C. M., Carter, C. D., and Meier, W., "Highspeed Laser Diagnostics for the Study of Flame Dynamics in a Lean Premixed Gas Turbine Model Combustor," Experiments in Fluids, Vol. 52, No. 3, 2012, pp. 555-567.

${ }^{23}$ Boxx, I., Carter, C. D., Stöhr, M., and Meier, W., "Study of the mechanisms for flame stabilization in gas turbine model combustors using kHz laser diagnostics," Experiments in Fluids, Vol. 54, No. 5, 2013, p. 1532. 